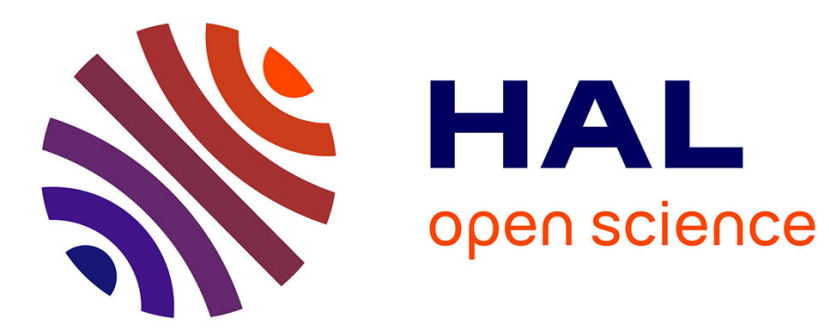

\title{
Interval Analysis and Reliability in Robotics
}

Jean-Pierre Merlet

\section{To cite this version:}

Jean-Pierre Merlet. Interval Analysis and Reliability in Robotics. 2006. inria-00001152

\section{HAL Id: inria-00001152 \\ https://hal.inria.fr/inria-00001152}

Submitted on 15 Mar 2006

HAL is a multi-disciplinary open access archive for the deposit and dissemination of scientific research documents, whether they are published or not. The documents may come from teaching and research institutions in France or abroad, or from public or private research centers.
L'archive ouverte pluridisciplinaire HAL, est destinée au dépôt et à la diffusion de documents scientifiques de niveau recherche, publiés ou non, émanant des établissements d'enseignement et de recherche français ou étrangers, des laboratoires publics ou privés. 


\title{
Interval Analysis and Reliability in Robotics
}

\author{
J-P. Merlet \\ INRIA, Sophia-Antipolis, France
}

\begin{abstract}
A robot is typical of systems that are inherently submitted to uncertainties although they should be highly reliable (i.e. for a robot used in surgical applications). The sources of uncertainties are the manufacturing tolerances of the mechanical parts constituting the robot which make the real robot always different from its theoretical model and control errors. We exhibit properties of a robot that are sensitive to the uncertainties and we present methods, using mainly interval analysis, that allow one to manage these uncertainties to ensure the reliability of the robot.
\end{abstract}

Keywords: interval analysis, robotics

Biographical notes: Will be provided later on

\section{INTRODUCTION}

A robot is basically a mechanical system whose main task is to move object or itself according to the decision of a high-level motion planner. Without losing to much generality will restrict ourself in this paper to industrial robot. These type of robots have a base that is attached to the ground and an end-effector that is charge of grasping and moving the objects. Hence the end-effector motion have to be controlled. An articulated mechanism connect the base and the end-effector. In the mechanism we have joints (such as revolute or prismatic joints denoted respectively $\mathrm{R}, \mathrm{P})$, some of which are actuated and are used to control the end-effector motion, while others are passive (i.e. they just follow the mechanism motion).

There are two main classes of industrial robots mechanical architecture: serial or parallel. In serial robot a successions of joint and rigid bodies leads from the base to the end-effector. Most industrial robot belong to this class, such as the Scara robot (figure 1) which is constituted of the succession of RRRP joints, all actuated, that allow to control 4 degrees of freedom of the robot (the 3 translations along the reference axis and one rotation around the $z$ axis). Another classical example of serial robot if the $6 \mathrm{R}$ robot constituted of a succession of 6 actuated revolute joints, that allows to control all 6 degrees of freedom of the end-effector. Most of 6 d.o.f. industrial robot have such architecture with the peculiarity that the axis of the last $3 \mathrm{R}$ joints are concurrent in the same point, for a reason that will be explained later on.

The second class of architecture is the parallel robot in which different kinematic chains connect the base to the end-effector. The most famous parallel robots is the Gough platform in which 6 articulated legs connect the base to

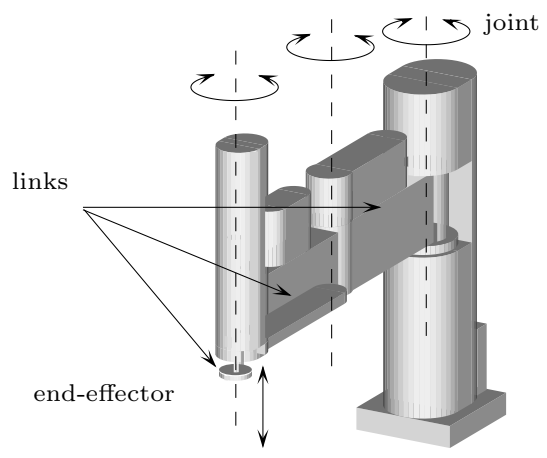

Figure 1: The SCARA serial robot, a robot allowing the control of 4 degrees of freedom of the end-effector

the platform (figure 2). Controlling the length of the 6 legs allows one to control all 6 degrees of freedom of the end-effector. Gough platforms are widely used for flight simulators. Let us introduce classical notation in robotics. The vector $\mathbf{X}$ is constituted of a set of parameters that define the values of the $n$ degrees of freedom of the endeffector. This vector is decomposed into the parameters $\mathbf{T}, \boldsymbol{\Gamma}$ that describes respectively the translation and orientation of the end-effector. In the most general case $\mathbf{X}$ is a 6 -dimensional vector, 3 of its components allowing to define the translation of the end-effector and the remaining 3 being angles allowing to define its orientation. The vectors $\Theta_{a}, \Theta_{p}$ define the value of respectively the actuated and passive joints of the robot (for example the rotation of a revolute joint). 


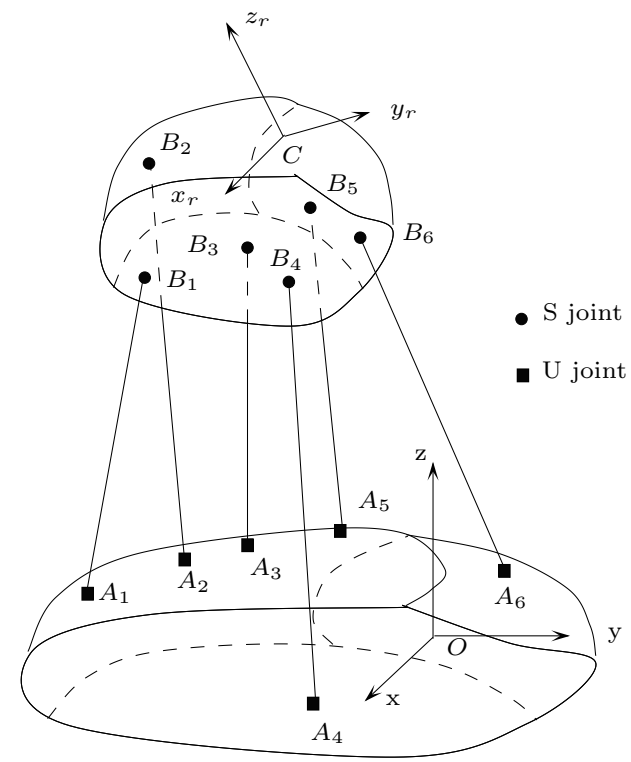

Figure 2: A parallel robot: the Gough platform. By changing the lengths of the 6 legs we may control the pose of the upper body.

\section{INTERVAL ANALYSIS}

As we will see many robotic problems may be safely solved by using methods based on interval analysis and we outline here the basic principle of this method (further details may be found in $(5 ; 8 ; 15 ; 16))$. Let $\mathbf{X}$ be a set of $n$ unknowns that are supposed to be included in ranges that defines a n-dimensional box $\mathcal{B}_{0}$. The problem to solve is to verify if a given property $P$ is satisfied for some $\mathbf{X}$ in $\mathcal{B}_{0}$. We have a decision operator $\mathcal{D}(\mathcal{B})$ that takes as input a subbox $\mathcal{B}$ of $\mathcal{B}_{0}$ and returns 1 if the property is satisfied over $\mathcal{B},-1$ if it is not satisfied and 0 if the property cannot be ascertained. Most of the decision operators that we will use will be based on interval arithmetics. This arithmetics allows to substitute variables by intervals in an expression and to get an interval for the value of the expression that is guaranteed to include all possible value of the expression whatever are the values of the variables in their respective ranges. Consider for example the expression

$$
x \cos (x)+y \sin (y)
$$

and assume that $x$ lie in the range $[0,1]$ and $y$ in the range $[1,2]$. The cosine of the range $[0,1]$ is the range $[0.54,1]$ while the sine of the range $[1,2]$ is $[\sin (1), 1]$. Hence the expression may be written as $[0,1] \times[0.54,1]+[1,2] \times$ $[\sin (1), 1]$. The first term is included in the range $[0,1]$ while the second term lies in the range $[\sin (1), 2]$. Consequently the whole expression may be evaluated as $[\sin (1), 3]$.

Hence interval arithmetics allows one to determine lower and upper bounds for a given function, being given ranges for the unknowns. The range provided by interval arithmetic is called an interval evaluation of the function. Note that the interval evaluation usually overestimates the real values of the maximum and minimum of a given function. For instance in the previous example the interval evaluation of $x \cos (x)$ for $x$ in $[0,1]$ has been calculated as $[0,1]$ through the result of the product of the 2 intervals $[0,1]$, $[0.54,1]$. But clearly there is no $x$ in $[0,1]$ such that $x \cos (x)$ is equal to 1 . Such overestimation is due to the multiple occurences of the same variable in the expression. When calculating the interval evaluation of $x \cos (x)$ we proceed in the same way than for the interval evaluation of $x \cos (z)$. However the amplitude of the overestimation will decrease with the width of the ranges and others methods may be used to calculate an interval evaluation with a lower overestimation.

An interesting property of interval arithmetics is that it can be implemented to take into account numerical roundoff errors due to the computers. A direct consequence is that the use of interval arithmetics allows to certify the decision even with respect to round-off errors. For example it may occur that the property cannot be ascertained even for fixed values of the unknowns as numerical errors in the computer arithmetics do not allow to certify the verification of the property and this case will be detected. However interval arithmetics has the drawback that its overestimation may leads to large computation time as numerous iterations may be necessary to ascertain the property.

In an interval analysis scheme sub-boxes of $\mathcal{B}_{0}$ will be stored in a list $\mathcal{L}$. Initially $\mathcal{L}$ has a single element $\mathcal{B}_{0}$ and sub-boxes will be added to the list during the algorithm: $m$ will denote the number of elements in the list at each iteration of the process. A typical interval analysis algorithm uses an index $i$ to indicate which box in $\mathcal{L}$ is processed, the index being initialized to 0 .

1. if $i>m$, then exit

2. if $\mathcal{D}\left(\mathcal{B}_{i}\right)=-1$, then $i=i+1$, go to step 1

3. if $\mathcal{D}\left(\mathcal{B}_{i}\right)=1$, then store $\mathcal{B}_{i}$ as a solution, $i=i+1$, go to step 1

4. if $\mathcal{D}\left(\mathcal{B}_{i}\right)=0$, then bisect one of the range of the box and add the two resulting boxes in the list, $m=m+2$, $i=i+1$, go to 1

We have described here only the very basic principle of the algorithm. Efficiency may be drastically improved, for example, by using a filter on the box $\mathcal{B}_{i}$ that allows to reduce its size or even discard the whole box (filters are usually specific of the solving problem). Example of such filter will be provided in some sections of this paper. Hence interval analysis cannot be considered as a "black box" and requires some expertise to determine the appropriate formulation of the problem and the right combination of filters to get the best efficiency. Note also that the basic scheme of interval analysis is appropriate for a distributed implementation: a master programm manages the list of boxes and send the boxes to slave computers that will run a few iterations of the solving scheme and then returns the result to the master computer. 
Interval analysis will be used in robotics because it allows to take into account the unavoidable uncertainties in the modeling of the robot or on its control. This means that we will always assume a uniform distribution of these uncertainties. This is motivated by two points:

- assuming a Gaussian distribution (or other type of distribution) for the uncertainties is not realistic for many mechanical parameters

- a uniform distribution allows to ensure that even in the worst case the robot will perform safely. This is critical for applications such as medical robotics

Classical interval analysis notation will be used:

- the lower (upper) bound of a range $x$ will be denoted $\underline{x}(\bar{x})$

- the mid point of a range $x$ is the real $(\underline{x}+\bar{x}) / 2$

- the width of a range $x$ is the real $\bar{x}-\underline{x}$

- the mid-point of a n-dimensional box is the the point whose coordinates are the mid-point of the ranges that constitute the box

We can now present examples of the use of this tool. For most of the problems presented in the next sections interval analysis is the only method that allows to solve safely the problem i.e. to obtain a guaranteed result. The algorithms presented in the next sections have been implemented using our our C++ library ALIAS $^{1}$ that uses the interval arithmetics BIAS/PROFIL and is interfaced with the symbolic computation software Maple.

\section{KINEMATICS}

A basic problem in robotics is to determine the relation between the actuated joint parameters and the pose of the end-effector. This is a crucial issue as the purpose of a robot is to allow to control the pose of the platform (i.e. the values of the elements of $\mathbf{X}$ ) through the control of the actuated joints (i.e. the values of the elements of $\boldsymbol{\Theta}_{\boldsymbol{a}}$. In the most general case a geometrical analysis of the mechanism allows one to establish an implicit relationship

$$
\mathbf{F}\left(\mathbf{X}, \Theta_{\mathbf{a}}, \Theta_{\mathbf{p}}, \mathcal{P}\right)=0
$$

where $\mathcal{P}$ denote elements characterizing the geometry of the robot as, for example, location of the axis of revolute joints, distances between two joints ... But this relationship should be simplified to generate more useful kinematic relations, namely the inverse and direct kinematics. The inverse kinematic $\mathbf{F}_{\mathbf{i}}$ gives directly the value of the actuated joints variables as functions of $\mathbf{X}$ while the purpose of the direct kinematic $\mathbf{F}_{\mathbf{d}}$ is to provide the value of $\mathbf{X}$ as functions of the actuated joints variables

$$
\Theta_{\boldsymbol{a}}=\mathbf{F}_{\mathbf{i}}(\mathbf{X}, \mathcal{P}) \quad \mathbf{X}=\mathbf{F}_{\mathbf{d}}\left(\boldsymbol{\Theta}_{\mathbf{a}}, \mathcal{P}\right)
$$

\footnotetext{
${ }^{1}$ www.inria.fr/coprin/logiciels/ALIAS/ALIAS.html
}

Note that in both cases the passive joint variables have been eliminated to get a direct relation between the input (i.e. usually the actuated joints variables) and the output of the system (the location of the end-effector of the robot). The usefulness of the inverse kinematic is clear: as one want to control $\mathbf{X}$ (i.e. a desired value of $\mathbf{X}$ is known) it is necessary to determine what should be the orders given to the motors (i.e. the $\boldsymbol{\Theta}_{\boldsymbol{a}}$ ) so that the end-effector reaches this pose. But the direct kinematics is also very useful as soon as one want not only to reach a given pose $\mathbf{X}_{\mathbf{1}}$ from another pose $\mathbf{X}_{\mathbf{2}}$, but also to do it by following a given trajectory (for example a line or a circle). The actuated joints variables $\boldsymbol{\Theta}_{\boldsymbol{a}}$ are measured by sensors and there are always uncertainties in the control. Hence the robot endeffector always deviates from its nominal trajectory and the direct kinematics is used to determine the amount of deviation, in order to correct it.

For serial robot the direct kinematic problem is usually easy to solve: indeed, starting from the base, the first joint variable allows to determine the location of the second joint and so on until we reach the end-effector. But usually the inverse problem (which amounts to solve the system of direct kinematic equations) may be difficult to solve. For example for the most general case of $6 \mathrm{R}$ robot it's only relatively recently that it was shown that the problem may have up to 16 solutions and that it was possible to exhibit an algorithm that is able to calculate them $(19 ; 24)$.

Reciprocally the inverse kinematic problem for parallel robots is usually simple: the locations of the extremities of the kinematic chain attached to the end-effector are known as soon as the location of the end-effector is given. It remains then to solve independently the inverse kinematics of each chain, and these chains are usually designed so that it is a simple task. For example for the Gough platform the actuated joint variables are the lengths of the legs, that can be calculated independently for each leg directly from $\mathbf{X}$, the solution being unique (see the Example section). On the other hand the direct kinematic is much more difficult and has usually multiple solutions. For the Gough platform it has been shown only recently that the problem may have up to 40 real solutions (i.e. for a given set of leg lengths there may be 40 possible poses for the end-effector) $(3 ; 7)$.

The inverse kinematics is basically used in a real-time context for the control of a robot. This is not a problem for parallel robots as they have usually a closed-form solution for this problem. But this may be problematic for serial robot such as the $6 \mathrm{R}$. To simplify drastically the problem most industrial robot uses a simple trick: the axes of the 3 last $\mathrm{R}$ joints are concurrent at the same point $C . \mathbf{X}$ is then defined as the coordinates of $C$ and the three angles representing the rotation around this point. In that case the last 3 joint angles control only the rotation while the 3 first one allows one to control the location of $C$ : the problem has been decoupled and this allows for a real-time computation.

Direct kinematics is also used for control purposes: this is not a problem for serial robot with their usually unique 
closed-form solution but is a difficulty for parallel robots. The direct kinematic problem has the following features:

1. it is crucial to determine the current pose i.e. the pose of the robot when the sensor data were measured and not another pose that is a solution of the direct kinematics

2. the direct kinematics is solved at regular sampling time of the controller. Hence, as the velocity of the end-effector is limited, we may define a neighborhood around the solution $\mathbf{X}_{\mathbf{k}-\mathbf{1}}$ obtained at time $t_{k-1}$ that should include the solution at time $t_{k}$. If more than one solution is found in this neighborhood, then the robot must be stopped as we are not able to determine the current pose

3. the neighborhood including the solution is not available when the robot is started. Hence all solutions should be determined so that the end-user may determine which is the current pose among all the solution poses (indeed determining numerically the current pose among the solutions is still an open problem). However in that case the real-time constraint may be relaxed

As mentioned earlier finding all solutions is difficult. Currently the fastest approach is based on the use of Gröbner basis (22), but an approach based on interval analysis is almost as fast (12). Current computation time ranges between a few seconds to one minute according to the geometry of the robot. Other methods have been proposed (mostly based on elimination or homotopy) but are not numerically safe.

The real-time solution of the problem is based on item 3: the pose $\mathbf{X}_{\mathbf{k}-\mathbf{1}}$ is usually used as an initial guess for a Newton-Raphson scheme. But such scheme is not safe: the method may not converge or worst converge to a solution that is not the current pose (and this may happen even if the initial guess is very close to the current pose). Furthermore this method does not allow to determine if there is more than one solution in the given neighborhood, which is crucial for a safe use of the robot. In that case interval analysis is very useful. Indeed the decision operator of the interval analysis scheme may use the Kantorovitch theorem (23), that is presented now.

Let a system of $n$ equations in $n$ unknowns:

$$
\mathbf{F}=\left\{F_{i}\left(x_{1}, \ldots, x_{n}\right)=0, i \in[1, n]\right\}
$$

Let $\mathbf{x}_{\mathbf{0}}$ be a point and $U=\left\{\mathbf{x} /\left\|\mathbf{x}-\mathbf{x}_{\mathbf{0}}\right\| \leq 2 B_{0}\right\}$, the norm being $\|A\|=\operatorname{Max}_{i} \sum_{j}\left|a_{i j}\right|$. Assume that $\mathbf{x}_{\mathbf{0}}$ is such that:

1. the Jacobian matrix of the system has an inverse $\boldsymbol{\Gamma}_{\mathbf{0}}$ at $\mathbf{x}_{\mathbf{0}}$ such that $\left\|\boldsymbol{\Gamma}_{\mathbf{0}}\right\| \leq A_{0}$

2. $\left\|\boldsymbol{\Gamma}_{\mathbf{0}} \mathbf{F}\left(\mathbf{x}_{\mathbf{0}}\right)\right\| \leq 2 B_{0}$

3. $\sum_{k=1}^{n}\left|\frac{\partial^{2} F_{i}(\mathbf{x})}{\partial x_{j} \partial x_{k}}\right| \leq C$ for $i, j=1, \ldots, n$ and $\mathbf{x} \in U$

4. the constants $A_{0}, B_{0}, C$ satisfy $2 n A_{0} B_{0} C \leq 1$
Then there is an unique solution of $\mathbf{F}=0$ in $U$ and Newton-Raphson iterative method used with $\mathbf{x}_{\mathbf{0}}$ as estimate of the solution will converge toward this solution. Conversely let compute $B_{1}$ as $1 /\left(2 n A_{0} C\right)$ and assume that $B_{1} \leq B_{0}$ : then there is a unique solution in the box $\left[x_{0}-2 B_{1}, x_{0}+2 B_{1}\right]$.

Hence Kantorovitch theorem allows one to establish a ball that includes one, and only one, solution. It is even possible to enlarge this box by using an inflation method based on the H-matrix theory of Neumaier $(16 ; 17)$. Assume that it has been determined that the ball $\left[x_{0}-\right.$ $\left.2 B_{1}, x_{0}+2 B_{1}\right]$ includes one solution $\mathcal{S}$ of $\mathbf{F}=0$. It may be shown that if the set $S_{\mathbf{J}}$ of Jacobian matrices of the system that are obtained in the ball $\left[x_{0}-2 B_{1}-\alpha, x_{0}+2 B_{1}+\alpha\right]$, where $\alpha$ is a positive constant, does not include any singular matrix, then this ball includes only $\mathcal{S}$ as solution of $\mathbf{F}=0$. Evidently calculating exactly the set $S_{\mathbf{J}}$ is difficult, but we may easily calculate a set of matrices that include $S_{\mathbf{J}}$. Indeed we may calculate an interval evaluation of each component of $\mathbf{J}$ over the ball $\left[x_{0}-2 B_{1}-\alpha, x_{0}+2 B_{1}+\alpha\right]$ and the resulting interval matrix defines a set of matrices that includes $S_{\mathbf{J}}$. We will mention in the Singularity section how to address the problem of checking the regularity of a set of matrices defined by an interval matrix but a possible method is to verify that the $n \times n$ interval matrix is diagonaly dominant, i.e. that

$$
\left|J_{i i}\right|>\sum_{j=1}^{j=n}\left|J_{i j}\right| j \neq i
$$

for all $i$ in $[1, n]$. This test may be used for an interval matrix and if it is diagonally dominant, then none of the matrices in the set is singular. In practice we start with a small $\alpha$ and increases it incrementally until the interval matrix is no more diagonally dominant.

The decision operator used for the direct kinematics solver returns -1 if the interval evaluation of at least one of the inverse kinematics equation does not include 0 . If a solution is found through the Kantorovitch theorem the search domain is reduced to its complementary part with respect to the ball that contains the solution.

Hence interval analysis allows one either to safely calculate the single solution in the neighborhood (i.e. to determine the current pose) or to determine that there is more than one solution in it.

\subsection{An example}

As an example we will consider the inverse kinematics of a Gough platform (figure 2). We define a reference $(O, \mathbf{x}, \mathbf{y}, \mathbf{z})$ that is attached to the base. A mobile frame $\left(C, \mathbf{x}_{\mathbf{r}}, \mathbf{y}_{\mathbf{r}}, \mathbf{z}_{\mathbf{r}}\right)$ is attached to the end-effector. The attachment points of the leg on the base (platform) are denoted by $A_{i}\left(B_{i}\right)$. The pose of the end-effector is defined by the following parameters:

- $x, y, z$ : the coordinates of the center $C$ of the endeffector in the reference frame 
- $\psi, \theta, \phi$ : three angles that describe the orientation of the end-effector. With these angles it is possible to calculate a rotation matrix $\mathbf{R}$ that transforms the components of a vector expressed in the mobile frame into its components in the reference frame. Typical of such angles are the Euler's angles with the rotation matrix

$$
\mathbf{R}=\left(\begin{array}{lll}
c_{\psi} c_{\phi}-s_{\psi} c_{\theta} s_{\phi} & -c_{\psi} s_{\phi}-s_{\psi} c_{\theta} c_{\phi} & s_{\psi} s_{\theta} \\
s_{\psi} c_{\phi}+c_{\psi} c_{\theta} s_{\phi} & -s_{\psi} s_{\phi}+c_{\psi} c_{\theta} c_{\phi} & -c_{\psi} s_{\theta} \\
s_{\theta} s_{\phi} & s_{\theta} c_{\phi} & c_{\theta}
\end{array}\right)
$$

where $c_{u}, s_{u}$ denote the cosine and sine of the angle $u$.

The calculation of the leg length is identical for each leg so that we can drop the indices. Basically this length is the Euclidean norm of the vector $\mathbf{A B}$. This vector may be written as

$$
\mathbf{A B}=\mathbf{A O}+\mathbf{O C}+\mathbf{C B}
$$

Being given the geometry of the robot the vector $\mathbf{A O}$ is known while the vector $\mathbf{O C}$ is an input of the problem. The vector $\mathbf{C B}$ may be written as $\mathbf{R} \mathbf{C} \mathbf{B}_{\mathbf{r}}$ where $\mathbf{C B}_{\mathbf{r}}$ denotes the coordinates of point $B$ in the mobile frame and is given by the geometry of the robot, while the rotation matrix $\mathbf{R}$ is an input of the problem. Hence the three vectors on the right hand side of the equation (3) may be calculated from the geometry and from the pose $\mathbf{X}$ of the robot, which allow to determine the vector $\mathbf{A B}$ and then the leg length.

As for the direct kinematics we have to solve the system of 6 non linear equations $\rho_{i}^{2}=\left\|\mathbf{A}_{\mathbf{i}} \mathbf{B}_{\mathbf{i}}\right\|^{2}$ derived from (3). Note however that other formulations may be used as well. For example we may choose as unknowns the 9 coordinates of three points $B_{i}$ (e.g. the coordinates of $\left.\mathbf{O B}_{1}, \mathbf{O B}_{2}, \mathbf{O B}_{3}\right)$. For a given geometry we have $\mathbf{O B}_{\mathbf{j}}=\sum_{k=1}^{k=3} \alpha_{k} \mathbf{O B}_{\mathbf{k}}$ for $j$ in $[4,6]$, where the $\alpha_{k}$ are constants which may be derived from the geometry of the end-effector. If $d_{i j}$ denotes the known distance between the points $B_{i}, B_{j}$ the constraint equations are

$$
\begin{array}{r}
\rho_{i}^{2}=\left\|\mathbf{A}_{\mathbf{i}} \mathbf{B}_{\mathbf{i}}\right\|^{2} \quad i \in[1,3] \\
d_{i j}^{2}=\left\|\mathbf{B}_{\mathbf{i}} \mathbf{O}+\mathbf{O B}_{\mathbf{j}}\right\|^{2}
\end{array}
$$

Equation (4) may be written as

$$
\left(x_{i}-a_{i}\right)^{2}+\left(y_{i}-b_{i}\right)^{2}+\left(z_{i}-c_{i}\right)^{2}=\rho_{i}^{2}
$$

where $a_{i}, b_{i}, c_{i}, \rho_{i}$ are known constants and $x_{i}, y_{i}, z_{i}$ are the unknown coordinates of the point $B_{i}$. The structure of this equation may be used to design a filter for an interval analysis method that will solve the direct kinematics. The equation may be written as

$$
\left(x_{i}-a_{i}\right)^{2}=\rho_{i}^{2}-\left(y_{i}-b_{i}\right)^{2}-\left(z_{i}-c_{i}\right)^{2}
$$

For a given box of the interval analysis algorithm we may compute an interval evaluation $\left[A_{i}, B_{i}\right]\left(\left[C_{i}, D_{i}\right]\right)$ of the left (right) hand side of the equation. If these intervals have no intersection (i.e. $A_{i}>D_{i}$ or $B_{i}<C_{i}$ ), then equation (5) has no solution and the box may be discarded. Now assume that the intersection $\left[U_{i}, V_{i}\right]$ of the two intervals is strictly included in $\left[A_{i}, B_{i}\right]$. The value of $x_{i}$ is then included in the ranges $\left[\sqrt{U_{i}}+a_{i}, \sqrt{V_{i}}+a_{i}\right],\left[-\sqrt{V_{i}}+a_{i},-\sqrt{U_{i}}+a_{i}\right]$, that may allow to update the range of this variable.

Consider for example the following equation:

$$
(x-1)^{2}+(y-4)^{2}+z^{2}=10
$$

with $x$ in $[3,4], y, z$ in $[1,2]$. We get $\left[A_{i}, B_{i}\right]=[4,9]$ and $\left[C_{i}, D_{i}\right]=10-[4,9]-[1,4]=[-3,5]$. The intersection of these ranges is $[4,5]$ and $x$ may be updated to $[3, \sqrt{5}+1] \approx$ [3, 3.236].

A similar process may be used for the variable $y_{i}, z_{i}$ and for each of the equations. For instance we write

$$
z^{2}=10-(x-1)^{2}-(y-4)^{2}
$$

with $x$ in the range $[3, \sqrt{5}+1], y, z$ in $[1,2]$. We get $\left[A_{i}, B_{i}\right]=[1,4]$ and $\left[C_{i}, D_{i}\right]=10-[4,5]-[4,9]=[-4,2]$ with an intersection $[1,2]$. The range for $z$ may thus be reduced to $[1, \sqrt{2}]$.

Such filter is called local as it uses only one of the equations of the system at the same time. There are also global filter that uses either a subset or the whole set of equations.

The structure of the equations (4) leads also to interesting properties (12):

- it allows to demonstrate a stronger version of the Kantorovitch theorem where the number $n$ of equations may be substituted by 3 . Hence the ball that includes a single solution of the system will be larger and as this ball is excluded form the search domain we get a faster algorithm

- the largest value of the constant $\alpha$ of the inflation method of Neumaier may be calculated formally instead of incrementally, this leading to a faster determination of the ball with a single solution

\section{KINETICS, ACCURACY AND STATICS}

The purpose of kinetics to study the relation between the joint velocities $\dot{\Theta}$ and the end-effector velocities. The endeffector velocities can be decomposed into translation velocities $\dot{\mathbf{T}}$ and angular velocities $\boldsymbol{\Omega}$. Note that for robot involving rotations of the end-effector there is no orientation representation $\boldsymbol{\Gamma}$ whose derivatives are the angular velocities $\boldsymbol{\Omega}$. But we have $\boldsymbol{\Omega}=\mathbf{H}(\boldsymbol{\Gamma}) \dot{\boldsymbol{\Gamma}}$ where $\mathbf{H}$ is a matrix that is never singular (1). Hence we will use abusively the notation $\dot{\mathbf{X}}$ to denote the end-effector velocities vector. Derivation of the kinematic relations allows to establish the kinetic relation

$$
\mathbf{A} \dot{\mathbf{X}}+\mathbf{B} \dot{\Theta}=0
$$

As for kinematics we may define the inverse and direct kinetic relations as

$$
\dot{\mathbf{X}}=\mathbf{J}(\mathbf{X}, \boldsymbol{\Theta}, \mathcal{P}) \dot{\boldsymbol{\Theta}} \quad \dot{\Theta}=\mathbf{J}^{-1}(\mathbf{X}, \boldsymbol{\Theta}, \mathcal{P}) \dot{\mathbf{X}}
$$


where $\mathbf{J}$ is called the jacobian matrix of the robot, which is a function of the pose of the robot and of its geometry. Note that these relations may also be used to perform a first order analysis of the accuracy of the robot. Indeed the sensors that are used to measure the joint variables are affected by bounded measurement errors $\Delta \Theta$. These errors will induce in turn a positioning error $\Delta \mathbf{X}$ for the end-effector as the control is using the measurement for adjusting the pose of the end-effector. The joint errors and the positioning errors are related by

$$
\Delta \mathbf{X}=\mathbf{J}(\mathbf{X}, \boldsymbol{\Theta}, \mathcal{P}) \Delta \boldsymbol{\Theta}
$$

There is a strong duality between kinetic and static analysis. If $\mathcal{F}$ denotes the forces/torques applied on the endeffector (that is usually called a wrench) and $\boldsymbol{\tau}$ the forces or torques in the actuated joints, then we have

$$
\boldsymbol{\tau}=\mathbf{J}^{\mathbf{T}}(\mathbf{X}, \boldsymbol{\Theta}, \mathcal{P}) \mathcal{F}
$$

where $\mathbf{J}^{\mathbf{T}}$ denotes the transpose of $\mathbf{J}$. As we can see kinetic and static analysis rely on the determination of the jacobian matrix and on its inverse. For serial robot a closedform for $\mathbf{J}$ is usually easy to obtain but a closed-form for its inverse may be difficult to get (or be too large to be useful), while for parallel robots a closed-form for $\mathbf{J}^{-\mathbf{1}}$ is usually easy to obtain while a closed-form for $\mathbf{J}$ is difficult to get. For example the inverse jacobian of the Gough platform has as rows the normalized Plücker vector of the line associated to the legs of the robot:

$$
\mathbf{J}^{-\mathbf{1}}=\left(\left(\frac{\mathbf{A}_{\mathbf{i}} \mathbf{B}_{\mathbf{i}}}{\left\|\mathbf{A}_{\mathbf{i}} \mathbf{B}_{\mathbf{i}}\right\|} \quad \frac{\mathbf{C B}_{\mathbf{i}} \times \mathbf{A}_{\mathbf{i}} \mathbf{B}_{\mathbf{i}}}{\left\|\mathbf{A}_{\mathbf{i}} \mathbf{B}_{\mathbf{i}}\right\|}\right)\right)
$$

Classical problems in robotics are related to equations (7-9). Let us assume that the end-effector will have to move within a known workspace $\mathcal{W}$, i.e. that $\mathbf{X}$ is constrained to lie within a closed n-dimensional variety. For example the location of the center of the end-effector will move within a sphere, while its rotation angles have to lie within some pre-defined ranges. The following problems have to be solved

- type 1: being given bounds on the actuated joints velocities, forces/torques, errors of the sensors, determine the maximal end-effector velocities, wrench, positioning errors

- type 2: being given bounds on the end-effector velocities, wrench, positioning errors, determine the maximal actuated joint velocities, forces/torques, sensors errors

for any $\mathbf{X}$ in the workspace $\mathcal{W}$. These problems are of the highest practical interest. A typical problem of type 1 may be illustrated for a medical robot. For such application the positioning accuracy of the robot, that will operate a real patient, is clearly of vital importance. When this type of robot is designed, joint sensors must be chosen so that the effects of the sensors measurement errors $\Delta \boldsymbol{\Theta}^{m}$ on the positioning errors of the robot are lower than a given threshold for any pose of the robot within the workspace in which it will have to operate: this may be called a verification problem. Hence we have to solve the following problem

$$
\text { find } \operatorname{Max}(\Delta \mathbf{X})=\mathbf{J}(\mathbf{X}, \boldsymbol{\Theta}, \mathcal{P}) \Delta \boldsymbol{\Theta} \quad \forall \mathbf{X} \in \mathcal{W}
$$

submitted to the constraint $|\Theta| \leq \Delta \Theta^{m}$. But a type 2 problem may also be derived from this equation. The cost of a sensor is highly dependent upon its accuracy and it is thus interesting to determine the maximal sensor error so that the maximal positioning errors of the end-effector are lower than given thresholds (this may be called the design problem). This may be formulated as follows:

$$
\text { find } \operatorname{Max}(\Delta \boldsymbol{\Theta})=\mathbf{J}^{-\mathbf{1}}(\mathbf{X}, \boldsymbol{\Theta}, \mathcal{P}) \Delta \mathbf{X} \quad \forall \mathbf{X} \in \mathcal{W}
$$

submitted to the constraints $\Delta\left|X_{m}\right| \leq X_{m}^{t}$ for all components of $\mathbf{X}, X_{m}^{t}$ being the threshold for the m-th component. Similar problems may be derived for the statics: verify that given actuated joints forces/torques allows to equilibrate a set of known wrenches or find the minimal joint forces/torques that will allow to equilibrate the wrenches for any pose of the end-effector within its workspace.

It may already be seen that for any type of robot one of these problems will be difficult to solve as either $\mathbf{J}$ or $\mathbf{J}^{-1}$ will not be known in closed-form. But any of these problems will be difficult as:

- we have to solve a global optimization problem and the globality of the maximum must be guaranteed

- there are uncertainties in the geometry parameters $\mathcal{P}$ and the optimization must take them into account

But there is another approach to the problem. Indeed it is not strictly necessary to determine exactly the maximum of the optimization problem. For example for the verification problem it is sufficient to show that the maximum of $\Delta \mathbf{X}$ is lower than the threshold. For the design problem not all values of the sensor errors are possible: indeed commercially available sensors provide only a finite set of possible accuracies, that may be ordered as $\left\{\Delta \boldsymbol{\Theta}^{0}, \Delta \boldsymbol{\Theta}^{1}, \ldots, \Delta \boldsymbol{\Theta}^{l}\right\}$. Hence determining that the maximal sensor error is greater than $\Delta \boldsymbol{\Theta}^{k}$ and lower than $\Delta \boldsymbol{\Theta}^{k+1}$ is sufficient to choose $\Delta \boldsymbol{\Theta}^{k}$ as a safe value for the sensor error.

If we look at equation (7) is appears that we have a linear relationship between the unknowns and that the optimization problem $(11,12)$ may be stated in one of the following two forms:

1. being given a vector $\mathbf{Y}$, a known matrix $\mathbf{A}$ and $\mathbf{B}=$ $\mathbf{A}(\mathbf{X}, \mathcal{P}) \mathbf{Y}$, shows that the maximum, for all $\mathbf{X}$ in $\mathcal{W}$, of each component $B_{m}$ of $\mathbf{B}$ is lower than a threshold $B_{m}^{t}$

2. being given a vector $\mathbf{B}$, a known matrix $\mathbf{A}$ and $\mathbf{Y}$ such that $\mathbf{A}(\mathbf{X}, \mathcal{P}) \mathbf{Y}=\mathbf{B}$, shows that the maximum, for all $\mathbf{X}$ in $\mathcal{W}$, of each component $Y_{m}$ of $\mathbf{Y}$ is lower than a threshold $Y_{m}^{t}$ 
where $\mathbf{A}$ will be either the jacobian matrix or its inverse according to the problem or the robot. For the first problem the use of interval analysis is appropriate. Indeed being given ranges for $\mathbf{X}$, and even for the parameters in $\mathcal{P}$, interval arithmetics allows one to calculate an interval evaluation for $\mathbf{B}=\mathbf{A}(\mathbf{X}, \mathcal{P}) \mathbf{Y}$. We may then design a decision operator that returns -1 if the lower bound of the interval evaluation is greater than $B_{m}^{t}$ for all components of $\mathbf{B}$ or 1 if the upper bound is lower than $B_{m}^{t}$ for at least one component of $\mathbf{B}$. In the former case the part of the workspace corresponding to the range for $\mathbf{X}, \mathcal{P}$ will satisfy the constraints. In the later case at least one constraint will be violated.

The second problem is more difficult as it is implicit, but interval analysis is also appropriate. Indeed we have to consider the solutions of a set of linear systems and show that they are all included in a given bounding box. Assuming that $\mathbf{X}, \mathcal{P}$ may be defined by ranges, we may consider that $\mathbf{A}$ is an interval matrix $\mathbf{A}^{\mathbf{i}}$, i.e. a matrix with interval coefficients. Various methods for calculating a box that includes all solutions of all linear systems defined by any real matrix whose coefficients are included in the corresponding ranges of an interval matrix have been proposed $(9 ; 16 ; 21)$. These methods should however be adapted as $\mathbf{A}$ is not only an interval matrix but has the stronger structure of a parametric matrix. Hence not all real matrices in the set defined by $\mathbf{A}^{\mathbf{i}}$ will correspond to a jacobian or inverse jacobian. Consequently the bounding box that is computed by classical methods, which is already larger than the optimal bounding box, will also be usually larger than the optimal bounding box that may be obtained for the set of jacobian or inverse jacobian matrices. Possible approaches to improve these methods is to take into account the derivatives of the coefficients with respect to $\mathbf{X}, \mathcal{P}$ in the Gaussian elimination and to precondition $\mathbf{A}$ by pre- or post-multipliying it by a real matrix $\mathbf{K}$. It may be shown that pre-conditionning may be very efficient for jacobian matrices as soon as the multiplication AK (or KA) is first done symbolically in order to reduce the multiple occurrences of the unknowns (which is the main reason of the over-estimation of interval arithmetic), and then to plug-in the real coefficients of $\mathbf{K}$ to get the conditioned matrix.

\section{SINGULARITY}

Singularity analysis is a crucial problem, especially for parallel robots. It may be intuitively explained by using the static equilibrium equation (9). Assume that the wrench $\mathcal{F}$ is given and that the joint forces/torques $\boldsymbol{\tau}$ should be established. As this equation is linear each element of $\boldsymbol{\tau}$ may be calculated as a ratio whose denominator is the determinant $\left|\mathbf{J}^{-\mathbf{T}}\right|$ of the transpose of the inverse jacobian matrix. If this determinant is 0 and the numerator is not 0 , then the joint force/torque will go to infinity, thereby leading to a breakdown of the robot. A pose $\mathbf{X}$ of the determinant is equal to 0 is called a singular configura- tion of the robot and a parallel robot usually exhibit such configuration (for example the reader may figure out that for a Gough platform whose base and platform are planar the configuration in which the base and platform lie in the same plane is a singular configuration) and some industrial prototypes have suffered from a spectacular breakdown when they have come close to a singular configuration.

Hence clearly singularity should be avoided. This has to be done either for each trajectory performed by the robot (as will be seen in the next section) or for the whole workspace $\mathcal{W}$ of the robot.

Usually the inverse jacobian matrix is known in closedform and it is may be possible to calculate its determinant by using symbolic computation software (although it may be quite large). We have then to determine if this determinant may cancel within the workspace and the result should be guaranteed. There is a single method that has been proposed for this purpose (13). An arbitrary point $\mathbf{X}_{\mathbf{1}}$ is selected in $\mathcal{W}$ and the sign of its determinant is safely computed using an interval evaluation (without lack of generality we will assume that it is positive). The purpose of the algorithm is to determine if there may be a pose, or a set of poses, such that the determinant is negative. Indeed if this is the case, any path connecting one such pose and the pose $\mathbf{X}_{\mathbf{1}}$ should cross a singular configuration. At the opposite if no such pose is found, then the workspace is singularity-free. We may thus use an interval analysis scheme with a decision operator that is based on the interval evaluation of the determinant. If its lower bound is positive, then the operators return -1. If the upper bound of the evaluation is negative, the decision operators returns 1 and this shows that singularities are present in the workspace. If the lower bound is negative and the upper bound is positive the decision operator returns 0 . This algorithm stops either when all boxes have been processed (in which case $\mathcal{W}$ is singularity-free) or as soon as a box with a negative determinant has been found.

A drawback of this algorithm is that it is difficult to take into account uncertainties in the geometry parameters $\mathcal{P}$ of the robot. Indeed if these uncertainties are added, the size of the closed-form of the determinant is so large that it cannot be calculated (this is, for example, the case for the Gough platform). However having a closed-form for the determinant is not strictly necessary as an interval evaluation of the determinant may still be calculated by using classical linear algebra methods on the interval matrix that may be derived from the inverse jacobian. A closed-form has however the interest of providing a simplified form for the determinant with a reduced number of multiple occurrences of the unknowns and hence leads to a sharper interval evaluation of the determinant. Still, testing the regularity of an interval matrix is well known problem in interval analysis (10;9). One of the most efficient method has been proposed by Rohn (20).

We define the set $H$ as the set of all n-dimensional vector $\mathbf{h}$ whose components are either 1 or -1 . For a given box we denote by $\left[a_{i j}, \overline{a_{i j}}\right]$ the interval evaluation of the com- 
ponent $J_{i j}^{-1}$ of $\mathbf{J}^{-1}$ at the i-th row and $\mathrm{j}$-th column. Given two vectors $\mathbf{u}, \mathbf{v}$ of $H$, we then define the set of matrices $\mathbf{A}^{\mathbf{u v}}$ whose elements $A_{i j}^{\mathbf{u v}}$ are

$$
A_{i j}^{\mathbf{u v}}=\overline{a_{i j}} \text { if } u_{i} . v_{j}=-1, \underline{a_{i j}} \text { if } u_{i} . v_{j}=1
$$

These matrices have thus fixed numerical components corresponding to lower or upper bound of the interval $J_{i j}^{-1}$. There are $2^{2 n-1}$ such matrices since $\mathbf{A}^{\mathbf{u v}}=\mathbf{A}^{-\mathbf{u},-\mathbf{v}}$. If the determinant of all these matrices have the same sign, then all the matrices $\mathbf{A}^{\prime}$ whose components have a value within the interval evaluation of $J_{i j}^{-1}$ are regular. Hence for the $6 \times 6 \mathbf{J}^{-\mathbf{1}}$ of a Gough platform if the determinant of the 2048 matrices of $\mathbf{A}^{\mathbf{u v}}$ have the same sign, then all matrices in the set are regular.

But here again testing the regularity of the interval matrix that may be derived from the inverse jacobian is only a sufficient condition, but not a necessary one as all real matrices derived from the interval matrix do not correspond to inverse jacobian. Classicaly for parametric matrices $\mathbf{K}(\mathbf{X})$ Rohn test is not applied on the interval matrix directly, but on a pre-conditioned matrix $\mathbf{A K}$ where $\mathbf{A}$ is a real regular matrix. Usually this matrix is chosen as $\mathbf{K}^{-\mathbf{1}}(\operatorname{Mid}(\mathbf{X}))$ where $\operatorname{Mid}(\mathbf{X}$ is the mid-point vector of the ranges for $\mathbf{X}$ (the purpose being to get a matrix AK that is closer to the identity matrix). But for a parametric matrix is is better to first compute symbolically the pre-conditioned matrix and then to plug-in the value of the components of $\mathbf{A}$. The most efficient methods seems to use an interval analysis scheme with a decision operator based on a a symbolic pre-conditioning of the inverse jacobian matrix and apply Rohn's regularity test on the pre-conditioned matrix. Consider for example the parametric matrix

$$
\mathbf{K}=\left(\begin{array}{cc}
x & x \\
y & 2 y
\end{array}\right)
$$

where the unknowns $x, y$ are supposed to lie in the range $[1,2]$. The determinant of the matrix being $x y$ the set of parametric matrices does not include a singular matrix. The corresponding interval matrix is:

$$
\mathbf{K}_{I}=\left(\begin{array}{ll}
{[1,2]} & {[1,2]} \\
{[1,2]} & {[2,4]}
\end{array}\right)
$$

whose interval evaluation of the determinant is $[-2,7]$. We cannot expect the Rohn test to succeed as the singular matrix

$$
\mathbf{K}_{S}=\left(\begin{array}{ll}
1 & 2 \\
2 & 4
\end{array}\right)
$$

is included in $\mathbf{K}_{I}$. If we use a numerical pre-conditioning we get

$$
\mathbf{A}=\left(\begin{array}{cc}
4 / 3 & -2 / 3 \\
-2 / 3 & 2 / 3
\end{array}\right)
$$

and a pre-conditioned matrix

$$
\mathbf{A} \mathbf{K}_{I}=\left(\begin{array}{cc}
{[0,2]} & {[-4 / 3,4 / 3]} \\
{[-2 / 3,2 / 3]} & {[0,2]}
\end{array}\right)
$$

The interval evaluation of the determinant of $\mathbf{A} \mathbf{K}_{I}$ is $[-8 / 9,44 / 9]$, that does not allow to state that $\mathbf{K}$ is regular. Assume now that we assume that the components of $\mathbf{A}$ are $a_{i j}$. The symbolic pre-conditionned matrix $\mathbf{S}_{s}$ is

$$
\mathbf{S}_{s}=\left(\begin{array}{cc}
x\left(a_{11}+a_{21}\right) & x\left(a_{12}+a_{22}\right) \\
y\left(a_{11}+2 a_{21}\right) & y\left(a_{12}+2 a_{22}\right)
\end{array}\right)
$$

If we now plug-in the numerical values of the $a_{i j}$ (equation 16) in this matrix we get

$$
\mathbf{S}_{K}=\left(\begin{array}{cc}
2 x / 3 & 0 \\
0 & 2 y / 3
\end{array}\right)
$$

For the range $[1,2]$ we get the matrix

$$
\mathbf{S}_{K}=\left(\begin{array}{cc}
{[2 / 3,4 / 3]} & 0 \\
0 & {[2 / 3,4 / 3]}
\end{array}\right)
$$

and the interval evaluation of the determinant of this matrix is $[4 / 3,16 / 3]$ allowing to state that $\mathbf{K}$ is never singular.

Another method proposed by Popova for interval parametric matrices may also be useful in some cases (18). Combining all these methods has allowed to test the presence of singularity within the workspace of robots with a geometry that takes into account the manufacturing tolerances in a computation time that ranges from a few seconds to 10 minutes.

\section{TRAJECTORY VERIFICATION}

A robot is submitted to various mechanical constraints that does not allow it to perform arbitrary trajectories. For example the leg of a Gough platform have a minimum and maximum length and the mechanical joints on the base and platform allow only for a limited rotation of the legs, while singularity should be avoided. Typical mechanical constraints may be defined as

$$
\mathbf{F}(\mathbf{X}) \leq 0 \quad \mathbf{G}(\mathbf{X}) \neq 0
$$

We will see in the next section that we may deal with these constraints at the design stage (provided that a desired workspace $\mathcal{W}$ is defined) to ensure that the constraints are satisfied whatever is the pose of the robot within its workspace. But this is not sufficient as in some cases the robot has to perform trajectories that lie outside the prescribed workspace. Hence a software allowing to verify the constraints for an arbitrary trajectory is still necessary. Here again uncertainties should be taken into account. They may occur at two different levels

1. modeling: the geometry of the real robot differs from its theoretical model that is used to calculate $\mathbf{F}, \mathbf{G}$

2. control: the trajectory followed by the robot will not be exactly the desired one as

- the theoritical model is used by the control law 
- there are measurement errors in the internal sensors that are used to control the trajectory

- control errors will always occur

A generic motion verifier may be designed as soon as the trajectory may be described as a time function. Without lack of generality we may assume that each component $X_{i}$ of $\mathbf{X}$ may be written as an analytical function of the the time $T$, which lie in the range $[0,1]$. As soon as a parser allows to calculate an interval evaluation of the functions $\mathbf{F}, \mathbf{G}$ with respect to a time range we may always design a decision a generic decision operator that checks if these constraints are verified for the whole interval $[0,1]$ (such a parser has been proposed in (11)). Modeling uncertainties may be introduced as ranges in $\mathbf{F}, \mathbf{G}$ while control error, that may reasonably be bounded, are introduced as ucertainty ranges in the component of $\mathbf{X}$. Hence if the trajectory is sucessfully checked, then we may ensure that the trjaectory is safe, even in the worst case. Trials have shown that such a generic motion verifier may check realistic constraints in a few seconds, i.e. almost in real time.

But this approach may also be used to optimize the trajectory as will be shown in the example.

\subsection{Verification: an example}

Assume that a Gough platform has to follow a circular trajectory with radius $R$ in an horizontal plane at altitude $z=z_{c}$ with a constant orientation (the rotation matrix is the identity matrix). The trajectory may be defined as

$$
\begin{aligned}
& x=R \cos (2 \pi T)+\Delta x \\
& y=R \sin (2 \pi T)+\Delta y \\
& z=z_{c}+\Delta z
\end{aligned}
$$

where $\Delta x, y, z$ represent the control errors (for simplicity reasons we will assume that there is no errors on the orientation but they may be introduced as well). The square of the leg lengths may be computed as

$$
\begin{aligned}
\rho^{2}= & \left(R \cos (2 \pi T)+\Delta x-x_{a}+x_{b}-\Delta x_{a}+\Delta x_{b}\right)^{2} \\
& \left(R \sin (2 \pi T)+\Delta y-y_{a}+y_{b}-\Delta y_{a}+\Delta y_{b}\right)^{2} \\
& \left(z+\Delta z-z_{a}+z_{b}-\Delta z_{a}+\Delta z_{b}\right)^{2}
\end{aligned}
$$

where the coordinates with the $a(b)$ subscript represent the coordinates of $A_{i}\left(B_{i}\right)$ and the $\Delta$ the modeling errors for these coordinates. The constraints that should be satisfied is

$$
\rho_{\min }^{2} \leq \rho^{2} \leq \rho_{\max }^{2}
$$

for all legs and for any $T$ in the range [0,1]. Equation (24) may be written as the sum of the square of 3 terms $U_{j}, V_{j}, W_{j}$ and one of the constraint may be written as

$$
U_{j}^{2}+V_{j}^{2}+W_{j}^{2} \leq \rho_{\max }^{2}
$$

The structure of this inequality allows to design a filter for the interval analysis algorithm. Assume that there is a
$T$ such that the maximal leg length constraint is violated. The inequality may be written for example as

$$
U_{j}^{2}>\rho_{\max }^{2}-V_{j}^{2}-W_{j}^{2}
$$

Without going into the details it may be seen that the calculation of the interval evaluation of the right handside term may be used to eventually modify the interval evaluation of $U_{j}^{2}$. In turn this new interval evaluation may be used to update the range of the variable that appears in $U_{j}$ : we may thus create a filter that will speed up the processing.

\subsection{Optimization: an example}

Consider for example a Gough platform that is used as a milling machine (see figure 7). Although the robot has 6 degrees of freedom only 5 are necessary for the task at hand as the spindle provides the rotation around the platform normal (which corresponds to the angle $\phi$ of the Euler angles). The value of $\phi$ is hence free and may be used to optimize the trajectory. First of all we have to ensure that the trajectory is feasible i.e. that the constraints on the leg lengths are respected over the whole trajectory. If we assume that the trajectory is defined through analytical time function for the pose parameters, then the leg lengths can be established as function of the time $T$ and of $\phi$. For a given time the square of a leg length may be written as $A \cos \phi+B \sin \phi+C$ where $A, B, C$ are variables that depends only upon the geometry of the robot and upon the time. For a given leg and a given time the equations $\rho_{i}^{2}=\rho_{\min }^{2}, \rho_{i}^{2}=\rho_{\max }^{2}$ have generally 2 solutions in $\phi$. Hence there will one or two intervals for $\phi$ such that $\rho_{\text {min }}^{2} \leq$ $\rho_{i}^{2} \leq \rho_{\max }^{2}$

If they are uncertainties in the geometrical model of the robot we are still able to determine ranges for $\phi$ so that the leg lengths constraints will be satisfied. We may then consider that the trajectory should be singularity-free. At a given time the determinant of the inverse jacobian is a polynomial $P$ in $\sin \phi, \cos \phi$

$$
P=r_{j k} \sin ^{j} \phi \cos ^{k} \phi
$$

If we impose that the absolute value of $P$ should be greater than a given threshold $\epsilon_{S}$, then the structure of $P$ is such that there will be in general ranges for $\phi$ such that $P$ is greater than $\epsilon_{S}$, even if they are uncertainties in the coefficients $r_{j k}$.

At this point we have still some freedom for the choice of $\phi$ for a given time time $T$. In the considered application we may assume that the wrench applied by the tool on the robot on the robot is constant. A possible optimization choice is to minimize the maximal absolute value of the actuator forces $\boldsymbol{\tau}$, such choice allowing to reduce the wear on the actuator. The purpose of the algorithm is to produce a time-law for $\phi$ as a set of values $\phi(0) \ldots \phi(\Delta t), \phi(2 \Delta t), \ldots \phi(1)$ which may be used to control the machine. An initial value of $\Delta t$ is provided by the user but will be adjusted by the algorithm if necessary. 
A mix of the decision operators presented in the previous sections allows to determine ranges for $\phi$ such that in a given time range range $[T, T+\Delta t]$ the leg lengths constraints are satisfied and the trajectory singularity-free. If such range cannot be found we divide by $2 \Delta t$ until either at least one range is found or the algorithm establishes that the leg lengths constraints will be violated whatever the value of $T$ or that the value of the determinant will be lower than $\epsilon_{S}$ for some $T$ in the range.

If we assume that ranges for $\phi$ have been found for a given time range $\left[T_{1}, T_{2}\right]$ we will use another interval analysis algorithm to determine what is the value of $\phi$ that leads to $\tau_{m}$, the minimal maximal absolute values for all the forces $\tau$. However being given the uncertainties in the task $\tau_{m}$ cannot be established as a real value but as a range. As usual we will consider the worst case and try to minimize $\overline{\tau_{m}}$. Still it does not make sense to calculate exactly the minimal value $\tau_{M}$ of $\overline{\tau_{m}}$ so we will stop the calculation as soon as we can can ascertain that we have determined a value of $\phi$ such that $\overline{\tau_{m}}-\tau_{M} \leq \alpha$, where $\alpha$ is a threshold that is fixed by the end-user.

We have thus to solve a global optimization problem and classical methods of interval analysis may be used for that purpose (5). In our case we have to optimize a function of $\phi$ that can be derived from (9), while the parameter $T$ lie in a given range. Determining exactly the value of $\overline{\tau_{m}}$ is an optimization problem of type (11) and therefore relatively difficult as we do not have an explicit formulation of the function. But we may still use an interval analysis scheme with a decision operator that solve the linear interval system (i.e. determine a range that includes $\left[\underline{\tau_{m}}, \overline{\tau_{m}}\right]$ ) and uses the upper bound of this range as possible value for $\overline{\tau_{m}}$. A current value $\tau_{M}$ of $\overline{\tau_{m}}$ is maintained all along the algorithm. The decision operator returns

- -1 if $\left|\tau_{M}-\underline{\tau_{m}}\right| \leq \alpha$ or if $\underline{\tau_{m}}>\tau_{M}$

- -1 if $\overline{\tau_{m}}-\tau_{m}$ is lower than $\alpha$. In that case we set all interval variables to the mid-point of their ranges, solve numerically the linear system (9) and uses the result to eventually update $\tau_{M}$

- 0 otherwise

We have implemented this approach in a generic way (14) in the sense that the trajectory may be described with arbitrary analytical functions without having to change the kernel of the algorithm. For that purpose the parser presented in the previous section allows to calculate the necessary interval evaluations. Figure 3 present a typical result of the algorithm for a circular trajectory. On these plots are presented the maximal absolute values of the joint forces for various fixed values for $\phi$ together with the maximal absolute values of the joint forces obtained with our method. It may be be noted that even for this simple trajectory the optimization method allows for a significant decrease of the maximal forces.

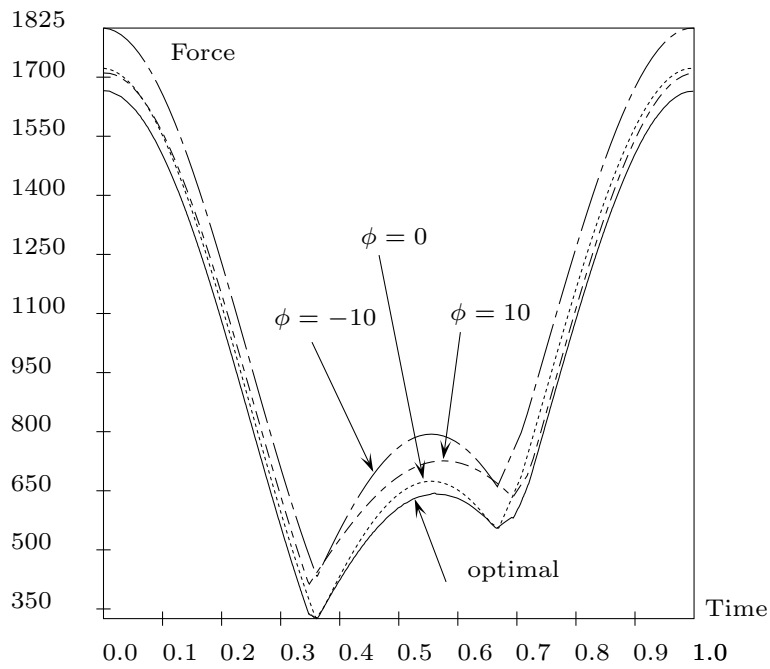

Figure 3: Maximal absolute values of the joint forces for various fixed value for $\phi$ and for our optimization method

\section{APPROPRIATE DESIGN}

The mechanical system that constitutes the basis of a robot should be designed so that a set of basic performance requirements $\left\{\mathcal{R}_{1}, \ldots, \mathcal{R}_{m}\right\}$ are satisfied, whatever is the pose of the robot in the desired workspace. For that purpose we have to choose theoretical values for the design parameters $\mathcal{P}$, keeping in mind that the values of the parameters for the real robot will alwyas differ from their theoretical values. A classical approach to satisfy the requirements is the trial-and-error methods that is based on a simulation software that allows to establish the performances of a given geometry. But this approach is difficult to use in practice as the cross-coupling between the influences of the various parameters is difficult to manage by hand as soon as the number of geometry parameters exceed a very small number. The classical academic way to deal with this problem is to use an optimal design methodology based on the cost-function approach (4). In this approach an index $I_{k}$ in the range $[0,1]$ is associated to each performance requirement $R_{k}$, the value of this index increasing with the level of the deviation of the performance with respect to the requirement. These indices are clearly functions of the design parameters $\mathcal{P}$ and a cost-function $\mathcal{C}$ is defined as

$$
\mathcal{C}=\sum_{k=1}^{k=m} w_{k} I_{k}(\mathcal{P})
$$

where $w_{k}$ are weights. Then a numerical optimisation procedure is used to find the value of $\mathcal{P}$ that minimizes the cost-function. There are numerous drawbacks to this approach:

- the definition of the indices $I_{k}$ are not easy for some performance requirement (e.g. the shape of the workspace of the robot)

- the use of a numerical optimisation approach imposes 
that the computation time for the calculation of the indices should be low. However we have seen in the previous sections that performance requirement such as accuracy or singularity detection require a significant computation time to be safely checked

- satisfaction of imperative requirements (e.g. requirements that must be satisfied by the robot) is difficult to strictly ensure.

- if they are antagonistic performance requirements (typically size of the workspace versus accuracy) the cost-function provides only a compromise between the requirements that is deeply influenced by the choice of the weights (2). Furthermore there is neither an intuitive choice for these weights nor a general method to find appropriate values for them if the design solution is not satisfactory

- uncertainties are difficult to take into account

Another approach is based on our practical experience of industrial robot design. The point is that in practice we have to deal with a list of requirements, some of them being more important than the others, but none having to be optimized per se. The objective become more to satisfy all of the requirements which led us to an appropriate design methodology.

If $\mathcal{P}$ is a m-dimensional vector we may define a parameter space in which each dimension is associated to one of the design parameters. One point in this space represents a unique robot design. Then it must be noted that the design parameters of a robot are usually bounded i.e. that only design solutions within some known ranges should be found. Hence values for the design parameters should be found within a m-dimensional box of the parameter space.

The idea of the appropriate design is simple. We will consider in turn each of the requirement $\mathcal{R}_{i}$ and assume that we are able to determine the region $\mathcal{Z}_{i}$ of the parameter space that defines all design parameter values for which the requirement $\mathcal{R}_{i}$ is satisfied.

A design that will satisfy all requirements has therefore a representative point in the parameter space that belongs to all $\mathcal{Z}_{i}$, hence to their intersection. If we assume that we can calculate the regions $\mathcal{Z}_{i}$ and then their intersection, then we will get all the design solutions. In practice however we cannot propose to an end-user an infinite set of design solutions. Thus the intersection will be sampled to provide a finite set of design solutions that are representative of various compromises between the different performances (including performances that may have not been included in the desired requirements).

But the principle of this design methodology may seem to be quite theoretical. Indeed computing the regions is clearly quite difficult in most cases and calculating the intersection is also a difficult step. But here uncertainties will play, for once, a positive role. Indeed computing exactly the regions for the requirements is not necessary because points of the parameter space that belong to a $\mathcal{Z}_{i}$ but are close to its border are only theoretical solutions: if they are chosen as nominal values for the design parameters, their values for the real robot may lead to a representative point in the parameter space that does not belong to $\mathcal{Z}_{i}$ and the requirement $\mathcal{R}_{i}$ will not be satisfied by the robot. Hence only an approximation of the $\mathcal{Z}_{i}$ is necessary. To simplify further the calculation of the $\mathcal{Z}_{i}$ we may use a relaxed version of the requirements provided that the full version of the requirements may be checked for the design solution. In that context we will see on a realistic example that interval analysis is an appropriate tool.

\subsection{An example}

We consider a Gough platform with planar base and platform. The 6 attachments points of the legs on the base are supposed to lie on a circle of radius $R_{1}$ with two adjacent points separated by an angle $\alpha$ (figure 4 ). The locations of the attachment points $A_{i}$ on the base is fully defined if $R_{1}, \alpha$ are known. Similarly the locations of the $B_{i}$ on the platform are fully defined by the radius $r_{1}$ of the platform and the angle $\beta$. The linear actuator in the leg

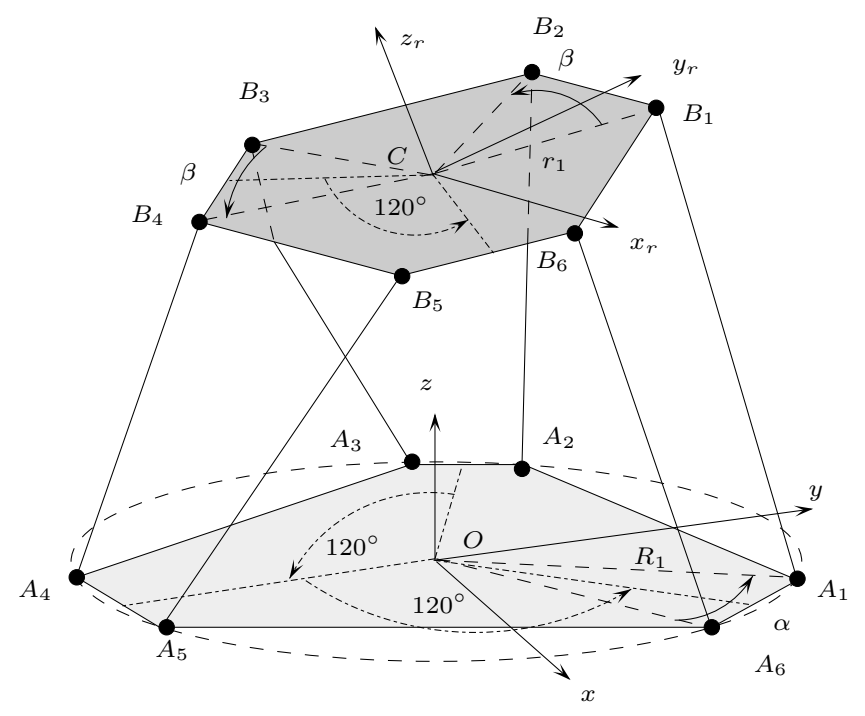

Figure 4: The design parameters for a Gough platform

have a stroke $S$ and the minimal length of the leg is $\rho_{\min }$. Hence the leg lengths $\rho$ are constrained to lie in the range $\left[\rho_{\text {min }}, \rho_{\text {min }}+S\right]$. Our set of design parameters $\mathcal{P}$ is defined as $R_{1}, \alpha, r_{1}, \beta, \rho_{\text {min }}, S$.

The requirement is that for any pose $(x, y, z, \psi, \theta, \phi)$ such that $x \in[\underline{x}, \bar{x}] y \in[\underline{y}, \bar{y}] z \in[\underline{z}, \bar{z}] \psi \in[\underline{\psi}, \bar{\psi}] \theta \in[\underline{\theta}, \bar{\theta}]$ $\phi \in[\underline{\phi}, \bar{\phi}]$, where the underlined and overlined quantities are known constants, the 6 leg lengths belong to the range $\left[\rho_{\text {min }}, \rho_{\text {min }}+S\right]$. In other words we have defined a 6-dimensional workspace $\mathcal{W}$ (which is an hyper-cube) for the robot and we require that any pose of $\mathcal{W}$ satisfies the constraint on the leg lengths.

We will relax somewhat the requirement for the design process by imposing that the leg lengths constraints are 
satisfied for the 64 corners $\left\{\mathbf{X}_{\mathbf{1}}, \ldots, \mathbf{X}_{\mathbf{6 4}}\right\}$ of the hypercube $\mathcal{W}$. As seen previously the square of the leg length $\rho$ can be computed as an analytical function of the pose $\mathbf{X}$ and of the design parameter $\mathcal{P}, F(\mathbf{X}, \mathcal{P})$. We will denote by $F_{i}=\left[F_{i}, \overline{F_{i}}\right]$ the interval evaluation of the length $\rho_{i}$ of the i-th leg.

All the design parameters may clearly be bounded. All of them should be positive, constraint on the overall size of the robot will give an upper bound for $R_{1}$ while we should have $r_{1}<R_{1}$ and symmetry constraints impose an upper bound for $\alpha, \beta$. We will use an interval analysis algorithm applied on the set of design parameters and an underlined or overlined parameter will indicate lower or upper bound for the parameters in the current box that is processed in the algorithm. The decision operator of this interval analysis algorithm is defined as follows:

1. returns -1 if $\overline{\mathcal{P}_{i}}-\underline{\mathcal{P}_{i}}<\epsilon_{i}$, where $\epsilon_{i}$ is the manufacturing tolerances associated to the design parameter $\mathcal{P}_{i}$

2. returns 1 if $F_{i} \geq \overline{\rho_{\min }}$ and $\overline{F_{i}} \leq \frac{\rho_{\min }}{\mathrm{X}_{64}} \underline{S}$ for all $i \in[1,6]$ and all poses $\mathbf{X}$ in $\left\{\mathbf{X}_{\mathbf{1}}, \ldots, \overline{\mathbf{X}_{\mathbf{6 4}}}\right\}$

3. returns -1 if $\underline{F_{i}}>\overline{\rho_{\min }}+\bar{S}$ or $\overline{F_{i}}<\underline{\rho_{\min }}$ for at least one $i$ in $[1,6]$ and one pose in $\left\{\mathbf{X}_{\mathbf{1}}, \ldots, \mathbf{X}_{\mathbf{6 4}}\right\}$

4. returns 0 otherwise

Case 2 indicates that for all the 64 poses the constraints on the 6 leg lengths are satisfied, whatever are the values of the design parameters in their current range: this is clearly a design solution. This is even a robust design solution with respect to uncertainties: indeed if the current parameter range for $\mathcal{P}_{i}$ is $\mathcal{P}_{i}^{c}=\left[\underline{\mathcal{P}_{i}}, \overline{\mathcal{P}_{i}}\right]$, then we may choose as nominal value for $\mathcal{P}_{i}$ any value in $\left[\mathcal{P}_{i}+\epsilon_{i} / 2, \overline{\mathcal{P}_{i}}-\epsilon_{i} / 2\right]$ and ensure that for the real robot $\mathcal{P}_{i}$ lie in the range $\mathcal{P}_{i}^{c}$ and hence that it will satisfy the leg length constraints. On the contrary case 3 indicates that one leg constraints is violated whatever is the design parameters values in their current robot: no design solution can be found in the current box. Case 1 is the place where uncertainties are taken into account. There may be theoretical design solution in the current box but even if one of those solutions was found we cannot ensure that the representative point of the real robot will still lie in the current box, and hence that the leg constraints will be satisfied. The boxes eliminated at step 1 are clearly on the border of the region $\mathcal{Z}_{i}$ and the result provided by the algorithm constitutes an approximation of this region.

This algorithm has the following properties:

- easy intersection calculation: the result of the algorithm is a set of 6-dimensional boxes. If all regions $\mathcal{Z}_{i}$ are computed with a similar algorithms the intersection of the obtained regions is simple to calculate. Furthermore the region $\mathcal{Z}_{i}$ may be used as input for the calculation of the region $\mathcal{Z}_{i+1}$, so that the region obtained for the requirement $\mathcal{R}_{m}$ will be directly the intersection of all $\mathcal{Z}_{i}$
- quality of the approximation: indices may be defined to qualify the quality of the calculation of $\mathcal{Z}_{i}$. Let $V_{\text {in }}, V_{\text {ne }}, V_{\text {out }}$ be respectively the total volume of the approximation, of the boxes that are neglected in case 1 of the decision operator and of the boxes that are discarded (case 3). A relative quality index in the range [0,1] may be defined as $V_{i n} /\left(V_{i n}+V_{n e}\right)$ (the closer the index is to 1 , the better the approximation). An absolute quality index, also in the range [0,1], may be defined as $\left(V_{\text {in }}+V_{\text {out }} /\left(V_{\text {in }}+V_{\text {ne }}+V_{\text {out }}\right)\right.$.

- incremental calculation of $\mathcal{Z}_{i}$ : instead of using the manufacturing tolerances for $\epsilon$ we may start a run of the algorithm with larger values and store the neglected boxes of case 1 in a special file. The quality of the approximation (whose total volume will be $V_{i n}^{1}$ ) for this run may be calculated using the previous indices. If it is not satisfactory we may restart the algorithm with a lower $\epsilon$ and with as input the boxes that have been neglected at the previous run. The boxes that will be obtained during this run have a total volume $V_{i n}^{2}$ and will be added to the boxes obtained at the first run, leading to an approximation with a total volume $V_{i n}^{1}+V_{i n}^{2}$ while the total volume of the neglected boxes will be $V_{n e}^{2}$. Hence we may incrementally improve the quality of the approximation until it is satisfactory, thereby reducing the computation time.

Figure 5 shows a cross section in the $\alpha, \beta, R_{1}$ space of the volume that is obtained.

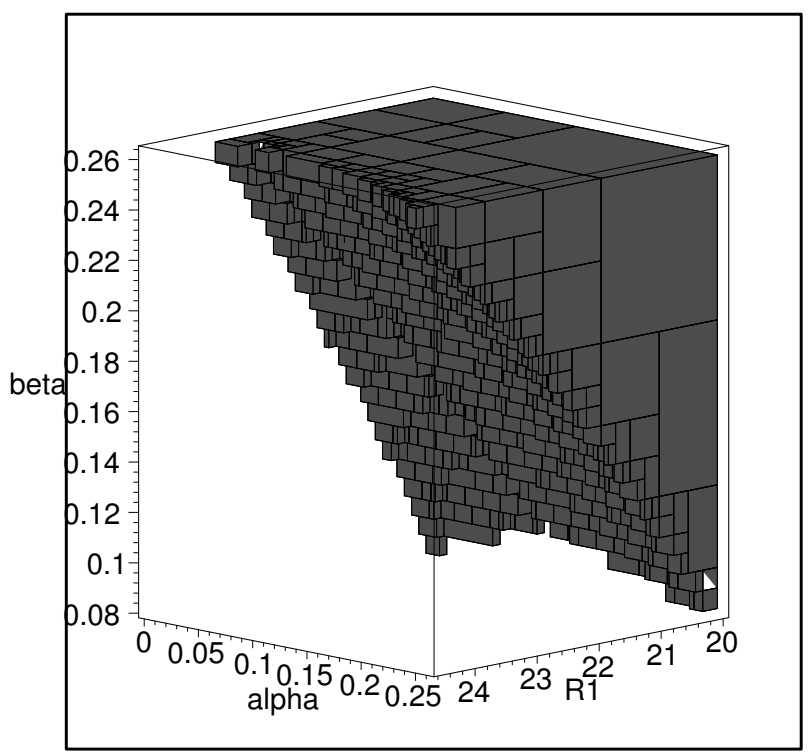

Figure 5: A cross-section in the $\alpha, \beta, R_{1}$ space of $\mathcal{Z}_{i}$. A point in this space represents a robot geometry and the workspace of this robot is guaranteed to include a set of 64 pre-defined poses of the end-effector

We have designed similar algorithms for accuracy and static analysis and this has allowed us to design successfully robots for various applications. For example a precise positionner with a measured absolute accuracy better than $1 \mu \mathrm{m}$ for a load of 2.5 tons has been desigggned for 


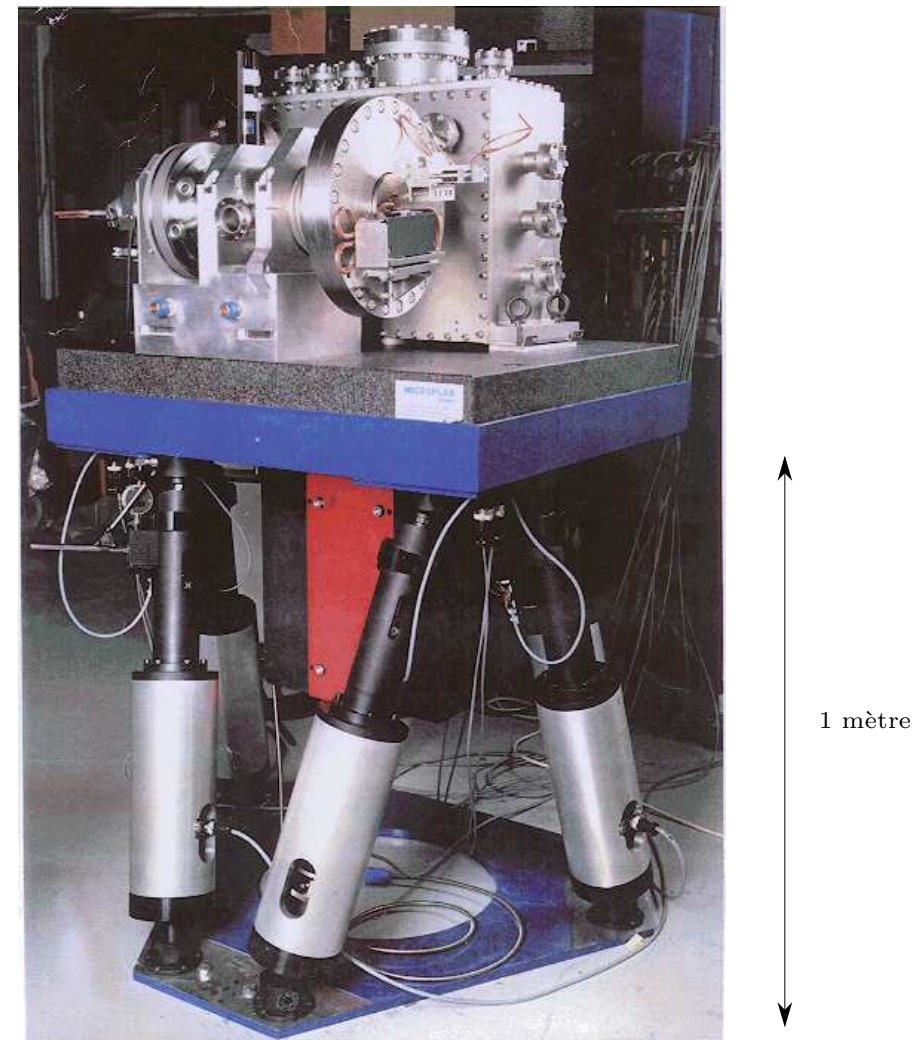

Figure 6: The positionner for the European Synchrotron Radiation Facility

tions Mécaniques des Vosges for the design of a high speed milling machine (figure 7). The interest of parallel robots for such application is the high rigidity and high velocities. A similar methodology has also been used for the design of a deployable space telescope for Alcatel (6) and for a surgical micro-robot (figure 8).

\section{CONCLUSIONS}

Robotics is typical as a field where uncertainties are unavoidable while the safety and reliability of the robot is crucial for many applications. Using statistical approaches to deal with these uncertainties is usually not possible because the safety should be ensured even in the worst case and because many of the uncertainties do not follow classical statistical distributions. We have shown that interval analysis is a tool that allows to deal with these uncertainties. But the efficient use of this tool requires a high level of expertise and a carefull analyis of the problem at hand.

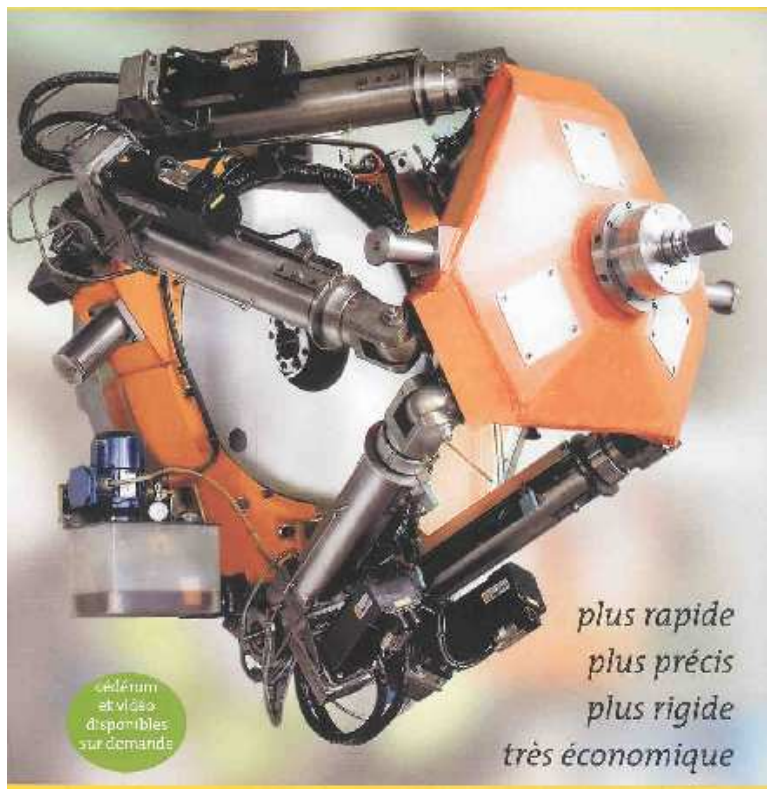

Figure 7: The high-speed milling machine of CMW

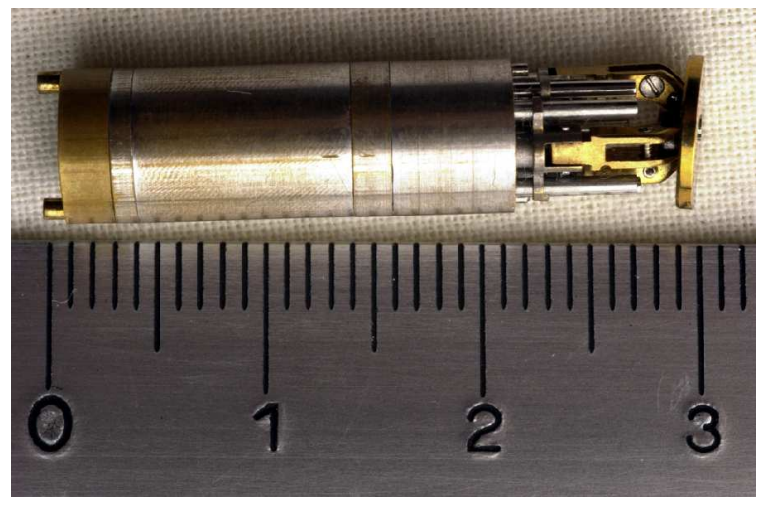

Figure 8: A surgical micro-robot

\section{ACKNOWLEDGEMENTS}


The work reported in this paper has been partially supported by the CNRS Robea program.

\section{REFERENCES}

[1] Angeles J. Fundamentals of robotic mechanical Systems. Springer, New-York, 1997.

[2] Das I. and Dennis J.E. A closer look at drawbacks of minimizing weighted sums of objectives for pareto set generation in multicriteria optimization problem. Structural Optimization, 14:63-69, 1997.

[3] Dietmaier P. The Stewart-Gough platform of general geometry can have 40 real postures. In $A R K$, pages 7-16, Strobl, June 29- July 4, 1998.

[4] Erdman A.G. Modern Kinematics. Wiley, New-York, 1993.

[5] Hansen E. Global optimization using interval analysis. Marcel Dekker, 1992.

[6] Hao F. and Merlet J-P. Multi-criteria optimal design of parallel manipulators based on interval analysis. Mechanism and Machine Theory, 40(2):151-171, February 2005.

[7] Husty M.L. An algorithm for solving the direct kinematic of Stewart-Gough-type platforms. Mechanism and Machine Theory, 31(4):365-380, May 1996.

[8] Jaulin L., Kieffer M., Didrit O., and Walter E. Applied Interval Analysis. Springer-Verlag, 2001.

[9] Kreinovich V. Optimal finite characterization of linear problems with inexact data. Research Report CS-0037, University of Texas at El Paso, 2000.

[10] Kreinovich V., Lakeyev A., Rohn J., and Kahl P. Computational complexity and feasibility of data processing and interval computations. Kluwer, 1998.

[11] Merlet J-P. A parser for the interval evaluation of analytical functions and its applications to engineering problems. J. Symbolic Computation, 31(4):475-486, 2001.

[12] Merlet J-P. Solving the forward kinematics of a Gough-type parallel manipulator with interval analysis. Int. J. of Robotics Research, 23(3):221-236, 2004.

[13] Merlet J-P. and Daney D. A formal-numerical approach to determine the presence of singularity within the workspace of a parallel robot. In F.C. Park C.C. Iurascu, editor, Computational Kinematics, pages 167-176. EJCK, Seoul, May, 20-22, 2001.
[14] Merlet J-P., Perng M-W., and Daney D. Optimal trajectory planning of a 5 -axis machine tool based on a 6-axis parallel manipulator. In $A R K$, pages $315-322$, Piran, June, 25-29, 2000.

[15] Moore R.E. Methods and Applications of Interval Analysis. SIAM Studies in Applied Mathematics, 1979.

[16] Neumaier A. Interval methods for systems of equations. Cambridge University Press, 1990.

[17] Neumaier A. Introduction to Numerical Analysis. Cambridge Univ. Press, 2001.

[18] Popova E.D. Strong regularity of parametric interval matrices. In Thirty Third Spring Conference of the Union of Bulgarian Mathematician, Borovets, April, 1, 2004.

[19] Raghavan M. and Roth B. Kinematic analysis of the 6R manipulator of general geometry. In 5th Int. Symp. of Robotics Research, pages 263-270, Tokyo, , 1990.

[20] Rex G. and Rohn J. Sufficient conditions for regularity and singularity of interval matrices. SIAM Journal on Matrix Analysis and Applications, 20(2):437-445, 1998.

[21] Rohn J. Systems of interval linear equations and inequalities (rectangular case). Research Report 875, Institute of Computer Science, Academy of Sciences of the Czech Republic, September 2002.

[22] Rouillier F. Real roots counting for some robotics problems. In J-P. Merlet B. Ravani, editor, Computational Kinematics, pages 73-82. Kluwer, 1995.

[23] Tapia R.A. The Kantorovitch theorem for Newton's method. American Mathematic Monthly, 78(1.ea):389-392, 1971.

[24] Wampler C. and Morgan A. Solving the 6R inverse position problem using a generic-case solution methodology. Mechanism and Machine Theory, 26(1):91-106, , 1991. 